\title{
Envenenamientos por mordedura de serpiente en Guatemala: revisión de literatura
}

\author{
Snakebite envenomings in Guatemala: literature review \\ Irmgardt A. Wellmann ${ }^{*}, 1,2$; Dennis Guerra-Centeno ${ }^{3}$ \\ ${ }^{1}$ Programa de Pós-Graduação em Medicina Tropical, Universidade do Estado do Amazonas. Manaus, Amazonas, Brasil; \\ ${ }^{2}$ Facultad de Ciencias Médicas; ${ }^{3}$ Instituto de Investigación en Ciencia Animal y Ecosalud, \\ Facultad de Medicina Veterinaria y Zootecnia, Universidad de San Carlos de Guatemala. Guatemala.
}

*Autor al que se dirige la correspondencia: irmgardtina@gmail.com

Recibido: 06 de agosto 2019 / Revisión: 11 de junio 2020 / Aceptado: 29 de julio 2020

\section{Resumen}

$\mathrm{E}$ 1 accidente ofídico es una enfermedad tropical desatendida que ocasiona un problema de salud pública en el mundo, siendo una de las principales causas de morbilidad y mortalidad en las áreas empobrecidas de América Latina. En Guatemala se distribuyen 23 especies de serpientes venenosas de importancia médica. La composición de los venenos es compleja y diversa, resultando en una variedad de manifestaciones clínicas. Los departamentos con mayor incidencia de ofidismo son Petén, Alta Verapaz, Quiché, Escuintla e Izabal. Estos accidentes afectan más a hombres que a mujeres, siendo la mayoría agricultores; el rango de edad más comprometido es de 10-19 años y principalmente ocurren en miembros inferiores. El tiempo medio que tarda la víctima en llegar al hospital es de $5.6 \mathrm{~h}$, siendo el retraso en la atención médica, un factor de riesgo para severidad y mortalidad. Los servicios de salud en ocasiones no cuentan con recursos idóneos para atender a las víctimas, brindando tratamiento sintomático. Simultáneamente, el tratamiento empírico tiene gran aceptación, sin embargo, los resultados de investigaciones realizadas con algunas plantas de uso común en casos de ofidismo, concluyeron que no es recomendable su uso aislado en el tratamiento del envenenamiento. Dada la falta de información se debe promover más investigación sobre el ofidismo en el país, siendo indispensable la elaboración de una ficha de reporte obligatoria. Además, deben elaborarse guías de tratamiento e identificación de serpientes para uso del personal médico, incluyendo programas de educación a nivel comunitario.

Palabras claves: Envenenamiento por serpiente, accidente ofídico, enfermedad desatendida, Bothrops asper, mordedura por serpiente

\section{Abstract}

nakebite envenoming is a neglected tropical disease that causes a public health problem in the world, being one of the main causes of morbidity and mortality in impoverished areas of Latin America. 23 species of poisonous snakes of medical importance are distributed in Guatemala. The composition of venoms is complex and diverse, resulting in a variety of clinical manifestations. The departments with the highest incidence of snakebites are Petén, Alta Verapaz, Quiché, Escuintla and Izabal. These accidents affect more men than women, the majority being farmers; the most compromised age range is 10-19 years and they mainly occur in the lower limbs. The average time it takes for the victim to reach the hospital is 5.6 hours, with delay in medical care being a risk factor for severity and mortality. Health services sometimes do not have adequate resources to care for victims, providing symptomatic treatment. Simultaneously, empirical treatment is widely accepted, however, the results of research carried out with some plants commonly used in cases of envenoming concluded that their isolated use is not recommended in the treatment. Given the lack of information, more research on snakebite envenoming in the country should be promoted, making the preparation of a mandatory report form essential. In addition, snake identification and treatment guides should be developed for use by medical personnel, including education programs at the community level. 


\section{Introducción}

El envenenamiento por mordedura de serpiente es una enfermedad tropical desatendida (World Health Organization [WHO], 2019) que resulta de la inyección de una secreción tóxica altamente especializada -el veneno- en algún tejido de un ser humano. Esta inyección ocurre a través de los colmillos (que son dientes modificados) que actúan a manera de agujas hipodérmicas y que están conectados con una glándula de veneno. Las circunstancias en que ocurre son accidentales, pudiendo generar alteraciones locales y sistémicas (Gutiérrez et al., 2017; Ministerio da Saúde, 2014).

Los accidentes ofídicos son un importante problema de salud pública en el mundo en vías de desarrollo. Es una de las principales causas de morbilidad y mortalidad, especialmente en las áreas empobrecidas de los trópicos y subtrópicos, como África subsahariana, el sudeste de Asia, Papúa Nueva Guinea y América Latina (Gutiérrez et al., 2017).

Se estima que al menos 421,000 envenenamientos y 20,000 muertes por mordeduras de serpientes ocurren anualmente en todo el mundo. Sobre la base de la estimación de que el número total de mordeduras de serpientes es de dos a tres veces el número de intoxicaciones, se estima que pueden ocurrir entre 1,200,000 y 5,500,000 accidentes ofídicos a nivel mundial. La mayoría de la carga de mordidas estimada se encuentra en el Sur y Sudeste de Asia, África Subsahariana y América Central y del Sur (Kasturiratne et al., 2008).

Siendo Latinoamérica una de las regiones de mayor prevalencia de casos de accidentes ofídicos, son escasos los esfuerzos de investigación de esta enfermedad y no suelen trascender el ámbito de la literatura gris, de tal manera que no contribuyen eficientemente con nuestro entendimiento del problema (Guerra-Centeno, $2018 b$ ), de igual forma los datos que pueden obtenerse sobre la mordedura por serpiente en Centroamérica, ya sea de publicaciones o de datos oficiales, no son completos y la magnitud real del problema es difícil de cuantificar (Bolaños, 1982). Específicamente, en Guatemala la información publicada en medios indexados es escasa (Guerra-Centeno, 2018b) y se estima que ocurren 600 envenenamientos por mordedura de serpiente al año, con incidencia de 4.2/100,000 habitantes al año (Gutiérrez, 2014).

\section{Contenido}

\section{Espcies de serpientes presentes en Guatemala y su distribución geográfica}

Guatemala es un país localizado en la región tropical, con características que le permiten poseer gran diversidad de fauna, dentro de la cual se encuentran alrededor de 138 especies de serpientes (Uetz et al., s. f.), de las que solo 23 son venenosas (Acevedo et al., 2010). Están divididas en ocho familias, pero en el país únicamente existen dos familias de serpientes venenosas de importancia médica para el humano, estas son:

- Familia Viperidae (víboras verdaderas y víboras de foseta): es la familia de mayor importancia médica por la cantidad de accidentes que causan y la pronta acción de su veneno. Dentro de esta familia se encuentran todas las víboras e incluye 15 especies de los géneros Bothrops (1), Agkistrodon (2), Atropoides (3), Crotalus (2), Cerrophidion (1), Bothriechis (4), Porthidium (2).

- Familia Elapidae (elápidos): en esta se ubican las siete especies de serpientes coral de Guatemala, del género Micrurus (7) y la víbora marina ( $H y$ drophis platurus) que habita en el océano Pacífico, hay muy pocos informes de mordeduras de esta especie en humanos, probablemente debido a limitaciones anatómicas y características de comportamiento. La incidencia de mordeduras de serpientes coral es baja, representando del 1 al $2 \%$ del número total de casos en la región centroamericana (Gutiérrez, 2009).

Algunas especies de otras familias presentan toxinas o venenos que no se consideran de importancia médica por la poca actividad y baja potencia de su veneno. Entre ellas se mencionan los géneros Conophis, Leptophis, Oxybelis, Trimorphodon y Thamnophis (Gutiérrez, 2009; Gutiérrez et al., 2017; Gutiérrez \& Sasa, 2002; Organización Panamericana de la Salud [OPS], 2009).

En nuestro país la mayoría de la población se dedica a la agricultura (Bouroncle et al., 2015; Instituto Nacional de Estadística, 2002); además, las condiciones climáticas han permitido que las serpientes venenosas se distribuyan a lo largo y ancho del territorio (Figura 1). Lo anterior da lugar a que las personas que viven en las áreas rurales y en las orillas de las zonas urbanas 

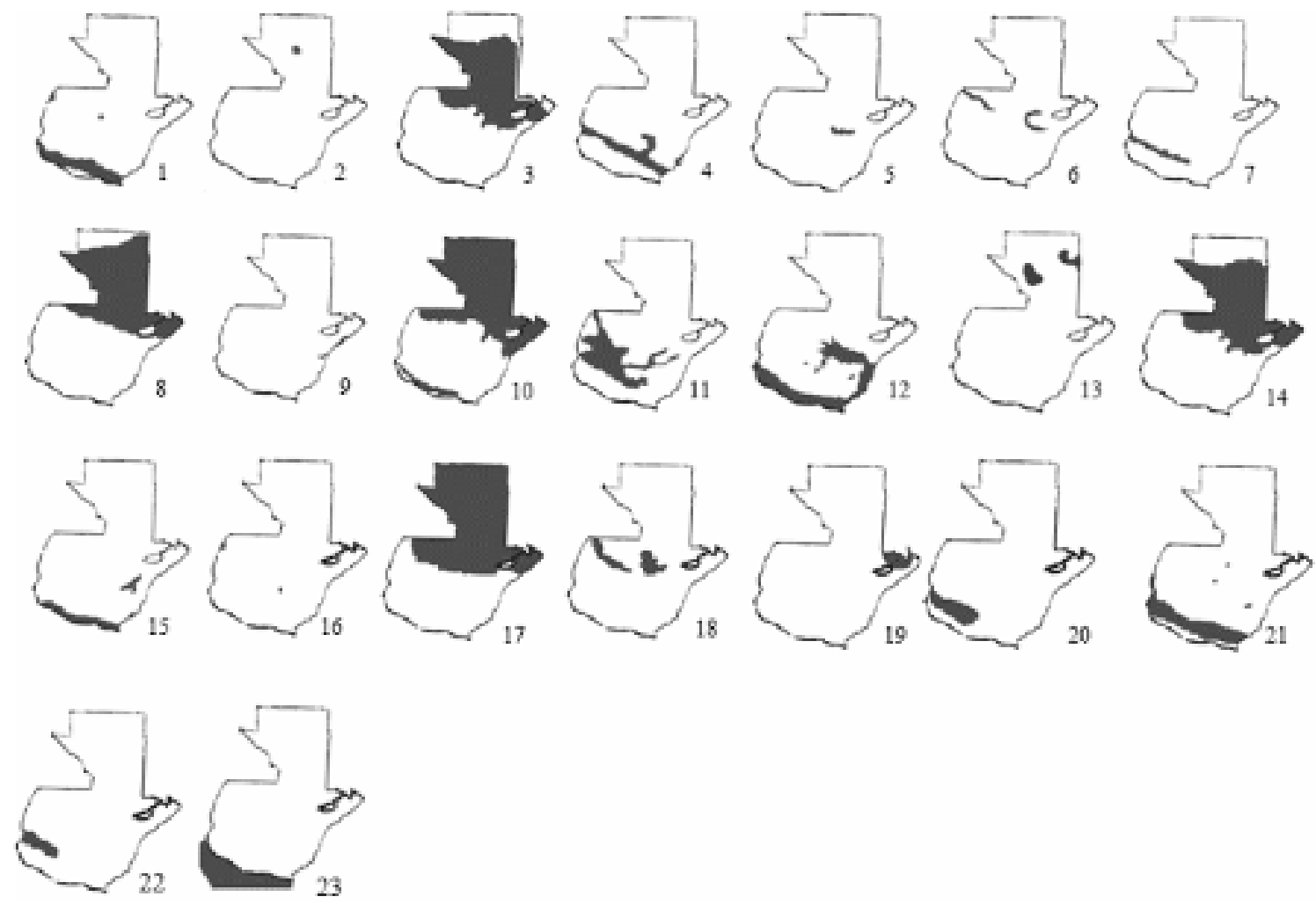

Nota. Distribución geográfica de las especies de serpientes venenosas de Guatemala

1) Agkistrodon bilineatus; 2) A. russeolus; 3) Atropoides mexicanus; 4) A. occiduus; 5) A. olmec; 6) Bothriechis aurifer; 7) B. bicolor; 8) B. schlegelii; 9) B. thalassinus; 10) Bothrops asper; 11) Cerrophidion godmani; 12) Crotalus simus; 13) C. tzabcan; 14) Porthidium nasutum; 15) P. ophryomegas; 16) Micrurus browni; 17) M. diastema; 18) M. elegans; 19) M. hippocrepis; 20) M. latifasciatus; 21) M. nigrocinctus; 22) M. stuarti; 23) Hydrophis platurus. (Acevedo et al., 2010; Guerra-Centeno, 2018b; Organización Panamericana de la Salud, 2009)

Figura 1. Distribución geográfica de las especies de serpientes venenosas de Guatemala

se encuentren en riesgo de sufrir accidentes por mordeduras de estos reptiles (OPS, 2009).

En el estudio realizado por Guerra-Centeno (2018b) se utilizaron datos oficiales del Sistema de Información Gerencial de Salud (Sigsa) del Ministerio de Salud Pública y Asistencia Social (MSPAS) para el período 2008-2013, observándose que no hay asociación significativa entre el número de especies de serpientes venenosas que ocurren por departamento y la morbilidad anual media por casos de accidente ofídico. Concluyendo que la ocurrencia de especies de serpientes venenosas es una condición suficiente más no determinante de los casos de accidente ofídico y que la morbilidad estaría entonces determinada por otros factores. Se sabe que el cambio de la cobertura vegetal y la perturbación humana típicos de cualquier agrosistema de uso múltiple, influencian la composición faunística del lugar. Por tanto la densidad de especies como $B O-$ throps asper y Boa constrictor podría incrementarse al aumentar la población de roedores en áreas de cultivos agrícolas (Guerra-Centeno et al., 2014). La distribución de serpientes venenosas se ha utilizado como un insumo para determinar el efecto del cambio climático en la carga de mordeduras de serpiente (Yañez-Arenas et al., 2016), y para determinar las poblaciones humanas vulnerables (Longbottom et al., 2018). El algoritmo propuesto por Bravo-Vega y colaboradores (2019), en Costa Rica, usa esas distribuciones para comprender la variación geográfica de la mordedura de serpiente, permitiendo así estimar la incidencia en lugares donde los datos son escasos. Y sus resultados subrayan la importancia de incorporar la abundancia, y no el nú- 
mero, de especies de serpientes de importancia médica como estimador de riesgo. Dicho modelo matemático sugiere que la distribución espacial de la mordedura de serpiente se debe principalmente a una combinación de factores ambientales que afectan la distribución de las especies venenosas y su asociación con el tamaño de la población humana expuesta (Bravo-Vega et al., 2019).

\section{Acciones de los venenos de las serpientes pre- sentes en Guatemala y aspectos clínicos de los accidentes ofídicos}

La composición de los venenos de serpiente es compleja y diversa, lo que resulta en un perfil bioquímico y toxicológico variable que determina una amplia gama de manifestaciones clínicas. Algunas toxinas en el veneno provocan daños en los tejidos locales, que a menudo resultan en secuelas permanentes, mientras que otras inducen efectos sistémicos, incluyendo manifestaciones neurotóxicas (que conducen, por ejemplo, a parálisis respiratoria, sangrado, lesión renal aguda y rabdomiólisis), cardiotoxicidad, hiperactividad autonómica o trombosis. Los venenos de las serpientes de la familia Viperidae causan efectos locales y manifestaciones sistémicas asociadas con sangrado, coagulopatías y shock hipovolémico. Los venenos de las serpientes de la familia Elapidae predominantemente inducen manifestaciones neurotóxicas, como la parálisis neuromuscular (Fatima \& Fatah, 2014; Gutiérrez et al., 2017; Hui Wen et al., 2015; Mackessy, 2009; McCleary \& Kini, 2013; Zamuner et al., 2005).

A pesar de que han sido publicadas clasificaciones de los efectos farmacológicos del envenenamiento, algunas bastante elaboradas, por razones prácticas los venenos de las serpientes centroamericanas pueden dividirse en solo dos grupos: (a) venenos que inducen manifestaciones locales (dolor, edema, hemorragia y necrosis) y efectos sistémicos (hemorragia, alteraciones de la coagulación y presión sanguínea y problemas renales); venenos de Crotalus, Agkistrodon y Bothrops pertenecen a esta categoría; y, (b) venenos neurotóxicos, típicamente los de coral y serpiente de mar (Bolaños, 1982).

En Guatemala se han desarrollado algunas investigaciones comparativas entre los venenos de las especies de Guatemala con especies de otros países de Centroamérica. Inicialmente, Overall (1987) realizó estudios de toxicidad de los venenos de B. asper y Bothrops nummifer (actualmente dividida en tres especies: Atropoides mexicanus, A. occiduus y A. olmec), com- parándolas con sus homólogas de Costa Rica, concluyendo que el veneno que $B$. asper de Guatemala es más tóxico que el de la de Costa Rica.

Más recientemente, Saravia y colaboradores (2001), analizaron el veneno de B. asper de Guatemala, demostrando que tiene actividades letales, hemorrágicas, miotóxicas, formadoras de edema, coagulantes, de desfibrinación y fosfolipasa A2 y muestra un perfil toxicológico similar al descrito previamente para $B$. asper de Costa Rica.

Chavéz (2001) caracterizó los efectos toxicológicos (letal, hemorrágico, coagulante y actividad fosfolipasa A2) del veneno de A. nummifer de Guatemala, revelando que este veneno presenta los efectos característicos de los vipéridos. El veneno de especímenes de Guatemala mostró ser menos letal, menos coagulante y menos hemorrágico que los venenos de $B$. asper y C. durissus durissus de especímenes de Guatemala y Costa Rica. Sin embargo, presentó importante actividad de la fosfolipasa A2 comparada con la descrita para las especies antes mencionadas.

Así mismo, Rojas y colaboradores (2001) realizaron un estudio comparativo en donde reportaron que los venenos de A. nummifer de Guatemala y Honduras tienen un perfil toxicológico cualitativamente similar, aunque hubo variaciones cuantitativas significativas en algunas de estas actividades entre los dos venenos.

Por su parte, Valdés y colaboradores (1994) reportaron que el veneno de la cascabel C. durissus durissus (actualmente renombrada y dividida en $C$. simus y $C$. tzabcan) de Guatemala es relativamente más letal que el de cascabel de Costa Rica, también se informó que dicha especie de Guatemala posee un efecto proteolítico que es incluso mayor que el presente en serpientes botrópicas de Costa Rica .

Se ha determinado que el veneno de $A$. bilineatus (actualmente dividida en A. bilineatus y A. russeolus) es casi 10 veces más hemorrágico y edematizante que el veneno de $B$. asper, siendo un veneno de alta vasculotoxicidad. Así también se ha reportado que no hay variaciones inmunoquímicas drásticas cuando se compara los venenos de B. asper de Costa Rica y Honduras, B. nummifer de Costa Rica y Honduras y $C$. d. durissus de Costa Rica y Guatemala (Rojas et al., 1987).

En cuanto a estudios realizados en la región centroamericana que describan los efectos in vivo que producen los venenos de especies nativas, podemos decir que existen pocos hasta la fecha. Partiendo del estudio realizado en Costa Rica por Barrantes y colaboradores (1985), con pacientes víctimas de accidente con $B$. asper, en donde se reportó que estos presentaron alte- 
raciones hemostáticas con evidencia de hipofibrinogenemia, aumento considerable en los niveles de los productos de degradación del fibrinógeno y disminución del plasminógeno, demostrando así que el componente fibrinolítico del veneno es considerable. Sin embargo, se destaca que uno de los retos continúa siendo la caracterización de las manifestaciones clínicas de los envenenamientos inducidos por varias serpientes en la región, con el objetivo de identificar patrones clínicos característicos de algunas especies (Gutiérrez, 2014).

En Guatemala, contamos con algunos datos de estudios descriptivos, Maltez (1994) recolectó información de los accidentes ofídicos atendidos del periodo de 1987-1992 en el departamento de Guatemala y reportó que $37 \%$ de los pacientes presentaron hipofibrinogenemia (fibrinógeno $<200 \mathrm{mg} / \mathrm{dl}$ ), 34\% prolongación del tiempo de protombina, $12 \%$ presentaron hematuria y $10 \%$ presentaron creatinina elevada.

Yee-Seuret y colaboradores (2012) realizaron un estudio en los departamentos de Huehuetenango y Petén, reportando que los síntomas locales fueron los predominantes en $92 \%(80 / 87)$ de los casos, seguidos de coagulopatías en $81.6 \%$ (trombocitopenia, prolongación del tiempo de sangrado, del tiempo de protrombina y del tiempo parcial de tromboplastina), así también informaron que los pacientes presentaron complicaciones como síndrome compartimental de extremidades (12.6\%), insuficiencia renal (8\%) y shock (3.4\%).

Las características clínicas más sobresalientes encontradas en otro estudio realizado en Petén, corresponden a hemorragia en sitios distantes a la herida en $24 \%$, coma en $14 \%$, vómitos en $16 \%$, deshidratación severa en $14 \%$, edema extenso y agudo en $22 \%$, flictenas y equimosis proximal y distal en $34 \%$, hipotensión en $30 \%$, anuria en $14 \%$ y mionecrosis en $8 \%$. Asimismo, se reportó que las patologías asociadas a la mortalidad por accidente ofídico fueron diabetes mellitus, alcoholismo crónico y anemia crónica. Además fue realizada necropsia en siete de los pacientes que fallecieron, reportándose en los hallazgos anatomopatológicos: hemorragia cerebral, necrosis renal y cirrosis hepática (esta última estaba presente en los pacientes que tenían antecedente de alcoholismo crónico) (Ramos, 1994). Cabe resaltar que el procedimiento estándar de oro para determinar las causas de muerte secundarias a las mordeduras de serpiente consiste en la realización de necropsias de diagnóstico, sin embargo estas se encuentran mal descritas en todo el mundo (da Silva Souza et al., 2018).

\section{Epidemiología de los accidentes ofídicos}

En Guatemala, el Centro Nacional de Epidemiología (CNE) del MSPAS es el encargado de llevar a cabo el registro y estadística de los accidentes por animales ponzoñosos que reportan los médicos y los hospitales (Letona, 2012).

Se cuenta con algunas investigaciones realizadas durante 1987-1993 en distintas localizaciones geográficas de Guatemala que aportan informaciones sobre las principales especies responsables de los accidentes ofídicos, sin embargo debe resaltarse que en la mayoría de los casos fue reportada como desconocida la especie de la serpiente, siendo muy pocos los casos en los que pudo ser identificada. Vemos así que $B$. asper fue la responsable de la mayoría de mordeduras en Petén Occidental (Mazariegos, 1993) e Izabal (García, 1993); A. bilineatus bilineatus (cantil de agua) en Amatitlán y sitios aledaños a este municipio (Palín y San Vicente Pacaya, Escuintla) (Mendoza, 1993), Chimaltenango (Barahona, 1993), Santa Rosa (Velásquez, 1993) y Sacatepéquez (López, 1993); y Porthidium ophryomegas у C. durisssus en El Progreso (Muralles, 1993).

En el estudio realizado por Letona (2012) donde utilizó la información estadística de la frecuencia de accidentes toxicológicos causados por animales ponzoñosos de Guatemala, reportó que las serpientes que han provocado la mayor cantidad de accidentes toxicológicos en el país son: B. asper, C. simus, M. nigrocinctus y A. mexicanus; y durante el período comprendido entre 2001 y 2010 se reportaron 7,377 mordeduras de serpientes por el CNE. Sin embargo, cabe mencionar que no se representa el total de casos ocurridos en esos años, debido a que el número anteriormente indicado solo hace ver aquellos casos que fueron reportados por los médicos de esos departamentos, pero en muchas ocasiones el paciente no llega a un hospital para ser atendido y, en su lugar, recibe tratamiento empírico en la comunidad o por chamanes o curanderos. Otras veces el paciente no logra recibir la atención en el momento y fallece, o bien el médico no sabe la razón de ingreso del paciente y por ende no lo reporta a las autoridades competentes como un ataque por animal ponzoñoso.

Posteriormente en un estudio realizado por Guerra-Centeno (2016) en las tierras bajas de Guatemala (Petén, Izabal, norte de Alta Verapaz y costa Sur de Guatemala), que es donde se distribuye la mayoría de las especies de serpientes venenosas del país, se analizaron 305 expedientes hospitalarios de casos de accidente ofídico correspondientes al período de 2008 
a 2013 en el Hospital Regional de Escuintla (HRE) y el Hospital Regional de San Benito, Petén (HRSBP), de los cuales 169 correspondieron al HRE y 136 al HRSBP. Se encontró que las especies que se consignaron en los expedientes con mayor frecuencia fueron $B$. asper en la región norte y C. simus en la región sur. Es importante resaltar que en ninguno de los 305 expedientes examinados se evidencia la confirmación de la especie de serpiente por un experto, a pesar de que muchas víctimas portan dicho animal al hospital; por lo tanto, en los expedientes se anota la especie que refiere el paciente (escrita como nombre común) o lo que señala el médico tratante.

Los accidentes ofídicos por serpientes del género Micrurus son muy escasos, teniendo publicados hasta la fecha únicamente el reporte de dos casos atendidos en un hospital de referencia de la ciudad de Guatemala en 2017, correspondientes a dos paciente de sexo masculino, el primero de 16 años referido de Petén y el otro de 41 años referido de Alta Verapaz, en ambos accidentes la serpiente fue identificada como Micrurus diastema debido a fotografías que portaban los pacientes (Castellanos \& Rodas, 2017).

\section{Características de los pacientes más afectados}

En una serie de investigaciones realizadas por estudiantes de medicina en varios departamentos del país se documentó que el rango de edad más afectado es el de 10-19 años (Barahona, 1993; González, 1994; López, 1993; Maltez, 1994; Mazariegos, 1993; Mendoza, 1993; Muralles, 1993; Ramos, 1994), coincidiendo con el estudio de Guerra-Centeno (2016) donde las edades con más casos comprendían de 11 a 20 años seguido por los grupos de 1 a 10 años y de 21 a 29 años, con edad media de 25.2 años (Guerra-Centeno, 2016).

Los accidentes ofídicos afectan más a hombres que a mujeres, así también se sabe que la mayoría de los afectados son agricultores (Barahona, 1993; García, 1993; Guerra-Centeno, 2016; López, 1993; Maltez, 1994; Mazariegos, 1993; Mendoza, 1993; Muralles, 1993; Ramos, 1994; Velásquez, 1993) por tanto los accidentes ocurren principalmente mientras la víctima se encuentra desempeñando alguna actividad agrícola. Sin embargo, el número de accidentes que ocurren mientras la víctima está en casa no es mucho menor (Guerra-Centeno, 2016).

En mujeres, las mordeduras acontecen cuando estas realizan trabajos domésticos, cuando contribuyen al sustento del hogar realizando labores agrícolas o cuando desempeñan actividades que implican un riesgo como la recolección de leña (Barahona, 1993; García, 1993; González, 1994; López, 1993; Maltez, 1994; Mazariegos, 1993; Mendoza, 1993; Muralles, 1993; Ramos, 1994; Velásquez, 1993) y agua, así como lavado de ropa a la orilla de los ríos (Barahona, 1993; Mendoza, 1993).

Los menores de edad que no tienen un oficio determinado, ayudan ocasionalmente a sus padres en las labores del campo o domésticas, lo que incluye recolección de leña en barrancos o cerros, pudiendo sufrir un accidente ofídico. En nuestro país también se ha reportado que un porcentaje de los afectados son estudiantes, entre los que se hallan adolescentes que fueron mordidos en sus ratos libres o cuando realizaban algún paseo (Barahona, 1993; García, 1993; González, 1994; Guerra-Centeno, 2016; López, 1993; Maltez, 1994; Mazariegos, 1993; Mendoza, 1993; Muralles, 1993; Ramos, 1994; Velásquez, 1993).

Con lo anterior, se evidencia que el solo hecho de vivir en el campo supone un riesgo de accidente ofídico incluso dentro de la casa. Esto último es propiciado por algunas prácticas agrícolas que son hostiles a las serpientes (como las quemas o la aplicación de insecticidas) y que las obligan a buscar refugio dentro de las casas de los campesinos, poniendo en riesgo principalmente a las amas de casa y a los niños (Guerra-Centeno, 2018b).

La incidencia de la mordedura a través del año es variable, no evidenciándose un patrón general, ya que fluctúa de acuerdo a las actividades particulares de cada región; en general, es mayor durante la preparación de los terrenos y la recolección de las cosechas (Bolaños, 1982).

\section{Localización anatómica}

En los datos recolectados en el país se conoce que la mayoría de las mordeduras ocurrieron en los miembros inferiores (Barahona, 1993; García, 1993; González, 1994; Guerra-Centeno, 2016; López, 1993; Mazariegos, 1993; Mendoza, 1993; Muralles, 1993; OPS, 2009; Velásquez, 1993).

Por otro lado, únicamente se cuenta con un estudio realizado en el departamento de Guatemala en donde se encontró que la mayoría de accidentes ocurrieron en miembros superiores y principalmente mano derecha (Maltez, 1994), debido a que son los miembros utilizados para el desarrollo de labores y recolección de cultivos (Guerra-Centeno, 2015b; Maltez, 1994; Yee-Seuret et al., 2012). 


\section{Localización geográfica}

En las investigaciones más grandes realizadas hasta la fecha, llevadas a cabo en distintos períodos y con distintas fuentes de información se reportó que los departamentos que presentaron mayor incidencia de ataques fueron: Alta Verapaz, El Progreso, Escuintla, Izabal, Petén y Quiché (Guerra-Centeno, 2018b; Letona, 2012).

La distribución geográfica de los casos del accidente ofídico, tiene una explicación ecológica y una sociológica. Desde el punto de vista ecológico, la morbilidad se explica por la distribución geográfica de las serpientes. Las especies venenosas se distribuyen principalmente en las tierras bajas del norte y del sur del país -donde ocurre la mayoría de los accidentes ofídicos-. Esto se debe a que las zonas de vida del norte y del sur (bosque húmedo subtropical y bosque muy húmedo subtropical), proveen las mejores condiciones ecológicas para las serpientes y, por lo tanto, es allí donde se concentra la mayoría de las especies (Guerra-Centeno, 2018b).

\section{Tratamiento médico}

No todas las mordeduras de serpientes causan envenenamiento, ya que algunas de ellas son causadas por serpientes no venenosas, e incluso en el caso de mordeduras por especies venenosas, varios casos no dan como resultado la inyección de veneno, lo que constituye mordeduras secas. Por lo tanto, el diagnóstico correcto de envenenamiento debe basarse en el análisis de los signos y síntomas objetivos presentados por el paciente, junto con los análisis de laboratorio realizados. Dicho examen clínico inicial, en lugar de la información proporcionada por la víctima o sus acompañantes, debe guiar el diagnóstico y las intervenciones terapéuticas posteriores (Gutiérrez, 2009; Letona, 2012; Ministerio da Saúde, 2001, 2019).

Después de un diagnóstico inicial, el pilar en el manejo clínico de estos pacientes es la administración intravenosa, ya sea de antiveneno polivalente (para mordeduras de víbora) o antiveneno anticoral (para mordeduras de serpiente coral), diluido en solución salina. El monitoreo cercano del paciente es crítico para detectar la aparición temprana de reacciones adversas a la terapia antiveneno, así como dar seguimiento de la evolución del caso. Además, debe considerarse la profilaxis antitetánica, junto con la administración de antibióticos en picaduras de víbora clasificadas como moderada a grave. Finalmente, se debe considerar el manejo de las complicaciones derivadas de los envenenamientos, dependiendo de las manifestaciones clínicas de cada caso (Gutiérrez, 2009).

La administración de antiveneno por vía intravenosa es el único tratamiento específico para neutralizar la intoxicación (Gutiérrez, 2019; Gutiérrez et al., 2017; Lomonte, 2012; Mackessy, 2009; WHO, 2019). También se utilizan analgésicos, soporte ventilatorio, fluidoterapia, hemodiálisis y antibióticos. Sin embargo, también se están explorando nuevas alternativas terapéuticas basadas en tecnologías de anticuerpos recombinantes y nuevos inhibidores de toxinas (Gutiérrez et al., 2017). La toma de decisiones para el manejo clínico se ve afectada por las incertidumbres sobre la identidad de la especie y la cantidad de veneno inyectado, así como la composición del mismo, ya que éste puede variar con la edad de la serpiente, su sexo y con la localización geográfica, aún en individuos de la misma especie (Amazonas et al., 2018; Daltry et al., 1996; Furtado et al., 2006; Gutiérrez et al., 2017; Menezes et al., 2006).

Se resalta el hecho que la única forma de atacar el accidente ofídico y contrarrestar sus efectos, es conocer los distintos venenos y sus mecanismos de acción, por lo que se requiere que en Guatemala se continúe con la caracterización de los venenos de las diversas serpientes y así formular sueros antiofídicos específicos para el país (Valdés et al., 1994), ya que aquí no se producen sueros antiofídicos y por lo tanto el Gobierno debe adquirirlos de proveedores que lo importan desde Argentina, México y Costa Rica. Lamentablemente esto repercute grandemente en el gasto, ya que el costo de cada dosis es de hasta 500.00 quetzales (62.50 USD) y por lo tanto el tratamiento de un solo paciente, podría ascender a 10,000.00 quetzales (1,250.00 USD). Además, éstos antivenenos presentan algunas particularidades ya que los provenientes de México y Costa Rica, son elaborados a partir de venenos de serpientes colectadas en esos países a diferencia de los importados de Argentina que son producidos con venenos de serpientes colectadas en Guatemala. Por tanto es posible, en el caso de los primeros, que la especificidad y, por consiguiente, la capacidad neutralizante de estos sueros, no sea la deseable para contrarrestar los efectos de los venenos de las serpientes autóctonas de Guatemala (Guerra-Centeno, 2015b).

Para evaluar dichos escenarios, han sido realizadas en el país muy pocas investigaciones, pudiendo citar el estudio realizado por Rojas y colaboradores 
(1987) donde se evaluó el antiveneno polivalente producido en Costa Rica por el Instituto Clodomiro Picado (ICP), el cual se prepara en caballos inmunizados con una mezcla de los venenos de $B$. asper, $C$. durissus $d u$ rissus y Lachesis muta (Lomonte, 2012). El objetivo fue evaluar la capacidad que tenía este antiveneno para neutralizar los efectos tóxicos y enzimáticos de los venenos de $A$. bilineatus y $C$. durissus durissus de Guatemala y de B. asper y A. nummifer de Honduras. Se concluyó que a excepción del efecto edematizante inducido por los venenos de $C . d$. durissus y $A$. nummifer, el antiveneno polivalente del ICP es efectivo en la neutralización de las actividades tóxicas y enzimáticas de estos cuatro venenos de Guatemala y Honduras. Es interesante que, pese a que el veneno de $A$. bilineatus no fue utilizado en la mezcla de inmunización para la elaboración del tratamiento antiofídico, este antiveneno polivalente fue efectivo en su neutralización, probablemente como consecuencia de las reacciones cruzadas, de tipo inmunológico existentes entre venenos (Rojas et al., 1987).

Posteriormente, se realizaron dos estudios que evaluaron la capacidad neutralizante del veneno de $A$. nummifer (mano de piedra) por los antivenenos polivalentes MYN (origen mexicano) y el costarricense del ICP, en el estudio realizado por Chávez (2001) se demostró que ambos antivenenos resultaron igualmente efectivos para neutralizar el efecto coagulante. No obstante, el antiveneno del IPC fue más efectivo que el antiveneno MYN para la neutralización de la actividad fosfolipasa A2 y fue el que neutralizó los efectos letal y hemorrágico; contrariamente al antiveneno MYN con el cual no se pudo alcanzar la neutralización de estos efectos.

El segundo estudio evaluó la capacidad de dichos antivenenos para A. nummifer de Guatemala y Honduras demostrando que el antiveneno ICP fue eficaz en la neutralización de las actividades letal, hemorrágica, miotóxica, coagulante, de desfibrinación y actividades de fosfolipasa A2, pero ineficaz contra la actividad formadora de edema. Por otro lado, el antiveneno MYN neutralizó las actividades hemorrágica, miotóxica, coagulante, de desfibrinación y actividades de fosfolipasa A2 aunque con una potencia menor que el del ICP. El antiveneno mexicano no logró neutralizar las actividades letales y formadoras de edema de los venenos de A. nummifer (Rojas et al., 2001).

Dado que $B$. asper es una de las especies que más accidentes ofídicos causa en el país, es indispensable conocer la capacidad de los antivenenos polivalentes MYN e ICP para neutralizar las actividades tóxicas del veneno. En el estudio realizado por Saravia y colabo- radores (2001), ambos antivenenos fueron efectivos contra todos los efectos estudiados, aunque el producto costarricense mostró una mayor potencia contra la mayoría de las actividades probadas y un mayor título de anticuerpos contra los componentes del veneno, según lo determinado por el inmunoensayo enzimático.

La información que se tiene en cuanto a la administración del suero antiofídico en el país, es también escasa, sin embargo en el estudio realizado por Maltez (1994), se reportó que en un hospital de referencia se administró antiveneno al 76\% de los casos, entre los motivos por los cuales no se aplicó se encuentran la falta de cumplimiento de criterios para su administración, el haber transcurrido mucho tiempo de evolución o por la falta de dicho tratamiento en el hospital en aquel momento.

En los casos de accidentes por $M$. diastema reportados por Castellanos y Rodas (2017), ambos recibieron tratamiento con neostigmina y presentaron buena evolución, a pesar de que en ninguno fue administrado el suero antiofídico específico debido a la inexistencia del mismo en el hospital de referencia donde fueron atendidos los pacientes.

Yee-Seuret y colaboradores (2012) reportaron que el tratamiento con antiveneno específico, administrado con prontitud y repetido las veces necesarias según la evolución clínica, controló $97.7 \%$ de los casos de envenenamiento, observándose que de los pacientes que presentaron alteraciones de la coagulación, el $45.5 \%$ se normalizaron en $48 \mathrm{~h}$. Lo anterior concuerda con lo publicado en Brasil sobre la importancia que tiene el tratamiento específico temprano en el desenlace del paciente, ya que se han reportado casos en donde el retraso en la administración del antiveneno ha complicado cuadros de envenenamiento botrópico con hemorragia intracraneana y secuelas a largo plazo para el paciente (Pardal et al., 2015; Pérez-Gómez et al., 2019). Recientemente, Silva de Oliveira y colaboradores (2019) reportaron que los niveles de veneno botrópico disminuyeron $12 \mathrm{~h}$ después de la terapia con antiveneno, además reportaron que los parámetros laboratoriales de la coagulopatía volvieron a los valores normales dentro de las $48 \mathrm{~h}$ posteriores a la sueroterapia y se mantuvieron hasta el alta.

Es interesante mencionar que una de las especies presentes en Guatemala, Porthidium nasutum (OPS, 2009), con distribución para el norte de Guatemala, es no procoagulante y no induce la desfibrin(ogen)ización in vivo, por lo tanto, no deben esperarse alteraciones en las pruebas de coagulación en pacientes mordidos por esta especie (Mackessy, 2009). 
Por otra parte, la terapia antiveneno también puede desencadenar reacciones adversas (Letona, 2012; Ministério da Saúde, 2019; Squaiella-Baptistão et al., 2018), en una investigación realizada en un hospital de referencia, se encontró que en pacientes con antecedentes médicos alérgicos, el 86\% (corresponde al 12\% del total de pacientes atendidos) presentó algún tipo de sensibilidad al suero antiofídico (Ramos, 1994).

En todas las investigaciones realizadas en el país se concluyó que la mayoría de servicios de salud no cuentan con los recursos necesarios para atender a la población que consulta por accidente ofídico y carecen de antivenenos por ser de alto costo, de difícil acceso y en ocasiones cuando si se obtienen, éstos no son específicos para las especies de nuestro país, por ser de origen extranjero, por lo que en varios centros asistenciales únicamente se brinda tratamiento sintomático a los pacientes (Barahona, 1993; García, 1993; González, 1994; Guerra-Centeno, 2015a; López, 1993; Maltez, 1994; Mazariegos, 1993; Mendoza, 1993; Muralles, 1993; Ramos, 1994; Velásquez, 1993).

\section{Tratamiento por medicina tradicional/ etnomedicina}

El tratamiento empírico-etnomédico, principalmente de emergencia, tiene gran aceptación entre la población afectada, debido esencialmente a las dificultades de transporte, de acceso a los servicios de salud y a la distancia entre las poblaciones, por lo que los pacientes utilizan los recursos disponibles en su localidad (curanderos, comadronas, hueseros, sobadores, boticarios) (Maltez, 1994), se sabe que en el país el tiempo medio que tarda la víctima en llegar al hospital es de 5.6 h (Guerra-Centeno, 2016) y el retraso en la atención médica se considera un factor de riesgo para severidad y mortalidad (Feitosa et al., 2015)

Existen algunos estudios científicos que demuestran la efectividad terapéutica de algunas de las plantas usadas en la medicina tradicional centroamericana para tratar el envenenamiento ofídico (Badilla et al., 2014; Coe \& Anderson, 2005; Giovannini \& Howes, 2017; Saravia-Otten et al., 2015, 2017).

En Guatemala, Saravia y colaboradores, 2015 realizaron dos estudios al respecto, en el primero se determinó la capacidad de los extractos de seis plantas de uso etnomédico (Acacia hindsii, Aristolochia maxima, Cissampelos pareira, Hamelia patens, Piper peltatum y Sansevieria hyacinthoides) para neutralizar los efectos proteolítico y fosfolipasa A2 (PLA2) del veneno de $B$. asper y en el segundo estudio, que fue una continuación, se evaluó la capacidad para inhibir el efecto coagulante que tenían dichas especies incluyendo otras cuatro especies más (Bursera simaruba, Eryngium foetidum, Hamelia patens, Pimenta dioica) (Saravia-Otten et al., 2017). Los resultados de ambas investigaciones concluyeron que no es recomendable el uso aislado de estas plantas en el tratamiento del envenenamiento por mordedura de $B$. asper, aunque posiblemente las que demostraron alguna actividad puedan resultar potenciadas al usarse en combinación con otras plantas, según la forma tradicional de preparación de los antídotos lo que podría potenciar su acción (Saravia-Otten et al., 2015, 2017).

Posteriormente, Chang (2017) evaluó la acción inhibitoria de los alcaloides de Sanseveira trifasciata y Tradescantia spathacea sobre el efecto hemolítico del veneno de $A$. mexicanus, concluyendo que es posible que los alcaloides de $T$. spathacea degraden las proteínas del veneno y recomienda realizar más estudios que evalúen la acción inhibitoria de dichos alcaloides en una mayor concentración y purificación.

\section{Medidas de prevención}

Es evidente que la mayoría de mordeduras ocurren en el pie, especialmente entre los campesinos, quienes usualmente trabajan descalzos ya sea por tradición o por razones económicas (Bolaños, 1982; Guerra-Centeno, 2015a) dato que clama por el uso de botas altas, zapatos o leggins de cuero cuando se viaja o trabaja en el campo; esto evita aproximadamente el $80 \%$ de los accidentes (Letona, 2012; Ministerio da Saúde, 2001).

Igualmente una proporción de accidentes ocurren en antebrazos, manos y dedos, como resultado directo del sistema agrícola utilizado en el área centroamericana, donde la mano es uno de los más importantes y activos instrumentos utilizados (Bolaños, 1982). Por lo tanto, se recomienda prestar atención al recolectar cultivos de árboles o arbustos, como el café, ya que algunas serpientes venenosas son arbóreas (Gutiérrez, 2009), además se indica el uso de guantes de cuero para manejar hojas secas, pilas de basura, leña, pajitas, etc., así como evitar introducir las manos en agujeros (Letona, 2012; Ministerio da Saúde, 2001).

Debido a que las serpientes se refugian en lugares cálidos, oscuros y húmedos, debe tenerse precaución al manipular pilas de leña, paja de frijol, maíz o caña (Ministerio da Saúde, 2001). 
Así también, se sabe que donde hay roedores hay serpientes, por lo que se recomienda la limpieza de los almacenes y patios, evitando el acaparamiento de basura, deben cerrarse agujeros en paredes y grietas en puertas, así como evitar la acumulación de escombros, piedras, ladrillos, baldosas, maderas, al igual que matorrales altos alrededor de las casas, pues estas condiciones atraen y albergan pequeños animales que sirven como alimento para las serpientes (Letona, 2012; Ministerio da Saúde, 2001).

\section{Recomendaciones}

La Organización Mundial de la Salud indica que deben promoverse prioridades en investigación para garantizar que las herramientas clínicas necesarias para abordar los accidentes ofídicos estén disponibles (WHO, 2019).

El problema del envenenamiento ofídico es un problema multidimensional, ya que el trabajador agrícola es vulnerable natural, física, económica, social, política, cultural, educativa, ecológica e institucionalmente. Se ha propuesto que la información generada por la epidemiología social, podría ser utilizada para concientizar al sector empresarial, al gobierno, a la academia y a los consumidores sobre la necesidad de lograr soluciones que contribuyan a disminuir las inequidades en salud y a mejorar la resiliencia y la calidad de vida de los trabajadores del agro y de los colectores de los productos silvícolas (Bouroncle et al., 2015; Guerra-Centeno, 2015b, 2018a).

Es indispensable la elaboración de una ficha oficial obligatoria de reporte por parte del MSPAS, para llevar un mejor control de los pacientes que ingresan a un centro hospitalario debido a un accidente ofídico (Letona, 2012), además se debe apoyar con orientación técnica y materiales de aprendizaje a los programas de educación para la mordedura de serpientes a nivel comunitario (WHO, 2019) ya que como se reportó en el estudio realizado por Valdés y colaboradores sólo el $24.8 \%$ del personal médico entrevistado posee un nivel de conocimiento aceptable sobre esta enfermedad (Valdés et al., 1994). Por lo tanto, se sugiere también la elaboración de guías de identificación de especie y tratamiento para el personal médico y paramédico de las distintas instituciones de salud en el territorio nacional.

Sin olvidar que es necesario garantizar la disponibilidad y accesibilidad de antivenenos en diferentes regiones del país y que estos se distribuyan siguiendo un plan de gestión que tome en cuenta las regiones con mayor incidencia de envenenamientos. Así como también debería ser considerado el acompañamiento médico de las personas que quedan con algún tipo de secuela física o psicológica luego del accidente ofídico ya que estas disminuyen su calidad de vida a largo plazo.

\section{Referencias}

Acevedo, M., Wilson, L. D., Cano, E. B., \& VásquezAlmazán, C. (2010). Diversity and conservation status of the Guatemalan herpetofauna.En Conservation of Mesoamerican amphibians and reptiles (pp. 406-435). Eagle Mountain Publishing.

Amazonas, D. R., Portes-Junior, J. A., Nishiyama-Jr, M. Y., Nicolau, C. A., Chalkidis, H. M., Mourão, R. H. V., ... Moura-da-Silva, A. M. (2018). Molecular mechanisms underlying intraspecific variation in snake venom. Journal of Proteomics, 181, 60-72. https://doi.org/10.1016/j.jprot.2018.03.032

Badilla, B., Chaves, F., Mora, G., \& Poveda, L. J. (2014). Edema induced by Bothrops asper (Squamata: Viperidae) snake venom and its inhibition by Costa Rican plant extracts. Revista de Biología Tropical, 54(2), 245. https://doi. org/10.15517/rbt.v54i2.13865

Barahona, G. A. (1993). Accidente ofídico: Estudio antropológico, clínico-epidemiológico, en el departamento de Chimaltenango, del 1 de enero de 1987 al 31 de diciembre de 1992. Guatemala (Tesis de licenciatura). Universidad de San Carlos de Guatemala, Guatemala.

Barrantes, A., Solís, V., \& Bolaños, R. (1985). Alteración de los mecanismos de la coagulación en el envenenamiento por Bothrops asper (Terciopelo). Toxicon, 23(3), 399-407. https:// doi.org/10.1016/0041-0101(85)90024-8

Bolaños, R. (1982). Las serpientes venenosas de Centroamérica y el problema del ofidismo. Primera parte. Aspectos zoológicos, epidemiológicos y biomédicos. Revista Costarricense de Ciencias Médicas, 3(2), 165-184.

Bouroncle, C., Imbach, P., Läderach, P., Rodríguez, B., Medellín, C., Fung, E., ... Donatti, C. I. (2015). La agricultura de Guatemala y el cambio climático: ¿Dónde están las prioridades para la adaptación? CGIAR Research Program on 
Climate Change. (Recuperado de http://hdl. handle.net/10568/45942

Bravo-Vega, C. A., Cordovez, J. M., Renjifo-Ibáñez, C., Santos-Vega, M., \& Sasa, M. (2019). Estimating snakebite incidence from mathematical models: A test in Costa Rica. PLOS Neglected Tropical Diseases, 13(12), e0007914. https://doi. org/10.1371/journal.pntd.0007914

Castellanos, E., \& Rodas, L. (2017). Manejo de envenenamiento micrúrico. Reporte de 2 casos. Revista del Colegio de Medicos y Cirujanos de Guatemala, 156(2), 79-80.

Chang J. E. (2017). Inhibición in vitro del efecto hemolítico del veneno de la serpiente mano de piedra (Atropoides mexicanus) causado por alcaloides de Sansevieria trifasciata y Tradescantia spathacea (Tesis de licenciatura). Universidad del Valle de Guatemala, Guatemala.

Chávez, J. J. (2001). Caracterización farmacopatológica del veneno de "mano de piedra», Atropoides nummifer (Ruppell, 1845, Squamata, Viperidae) y evaluación de la capacidad neutralizante de dos sueros antiofidicos polivalentes distribuidos en Guatemala (Tesis de licenciatura). Universidad de San Carlos de Guatemala, Guatemala.

Coe, F. G., \& Anderson, G. J. (2005). Snakebite ethnopharmacopoeia of eastern Nicaragua. Journal of Ethnopharmacology, 96(1-2), 303323. https://doi.org/10.1016/j.jep.2004.09.026

da Silva Souza, A., de Almeida, J., Alcântara, J. A., Freire, M., Alecrim, M. das G. C., Lacerda, M., ... Monteiro, W. M. (2018). Snakebites as cause of deaths in the Western Brazilian Amazon: Why and who dies? Deaths from snakebites in the Amazon. Toxicon, 145, 15-24. https://doi.org/10.1016/j. toxicon.2018.02.041

Daltry, J. C., Wüster, W., \& Thorpe, R. S. (1996). Diet and snake venom evolution. Nature, 379(6565), 537-540. https://doi.org/10.1038/379537a0

Pardal, P. P., Pinheiro, A. C., Silva, C. T., Santos, P. R., \& Gadelha, M. A. (2015). Hemorrhagic stroke in children caused by Bothrops marajoensis envenoming: a case report. Journal of Venomous Animals and Toxins including Tropical Diseases, 21(1), 53. https://doi.org/10.1186/s40409-015$0052-5$
Fatima, L-D., \& Fatah, C. (2014). Pathophysiological and Pharmacological Effects of Snake Venom Components: Molecular Targets. Journal of Clinical Toxicology, 4(2). https://doi. org/10.4172/2161-0495.1000190

Feitosa, E. L., Sampaio, V. S., Salinas, J. L., Queiroz, A. M., Da Silva, I. M., Gomes, A. A., ... Gutiérrez, J. M. (2015). Older age and time to medical assistance are associated with severity and mortality of snakebites in the Brazilian Amazon: A case-control study. PLOS ONE, 10(7), 1-15. https://doi.org/10.1371/journal.pone.0132237

Furtado, M. F. D., Travaglia-Cardoso, S. R., \& Rocha, M. M. T. (2006). Sexual dimorphism in venom of Bothrops jararaca(Serpentes: Viperidae). Toxicon, 48(4), 401-410. https://doi.org/10.1016/j. toxicon.2006.06.005

García, J. T. (1993). Accidente ofídico: Estudio antropológico, clínico-epidemiológico, en el departamento de Izabal del 1 de enero de 1987 al 31 de diciembre de 1992 (Tesis de licenciatura). Universidad de San Carlos de Guatemala, Guatemala.

Giovannini, P., \& Howes, M.-J. R. (2017). Medicinal plants used to treat snakebite in Central America: Review and assessment of scientific evidence. Journal of Ethnopharmacology, 199, 240-256. https://doi.org/10.1016/j.jep.2017.02.011

González, M. L. (1994). Accidente ofídico: Estudio clínico, antropológico y epidemiológico realizado en la Región Sur del departamento de Huehuetenango. 1ro. de enero de 1987 al 31 de diciembre de 1992, Guatemala (Tesis de licenciatura). Universidad de San Carlos de Guatemala, Guatemala.

Guerra-Centeno, D. (2015a). Epidemiología social del accidente ofídico en Guatemala: Hacia la comprensión de una enfermedad desatendida (Tesis de doctorado). Universidad Panamericana, Guatemala.

Guerra-Centeno, D. (2015b). Ética sobre el envenenamiento por serpiente en el agro paisaje de Guatemala. Ciencia, Tecnología y Salud, 2(1), 65-73.

Guerra-Centeno, D. (2016). Perfil epidemiológico del accidente ofídico en las tierras bajas de Guatemala. Ciencia, Tecnología y Salud, 3(2), 127-138. 
Guerra-Centeno, D. (2018a). Fenomenología del accidente ofídico: El significado de los daños físicos para la víctima. Revista Naturaleza, Sociedad y Ambiente, 5, 1-10.

Guerra-Centeno, D. (2018b). Morbilidad por accidente ofídico en Guatemala y su relación con la distribución geográfica de las especies de serpientes venenosas. Revista Electrónica de Veterinaria, 19(3), 31834.

Guerra-Centeno, D., Morán-Villatoro, D., FuentesRousselin, H., Meoño-Sánchez, E., \& ValdezSandoval, C. (2014). Herpetofaunal richness of Los Tarrales Natural Private Reserve, Atitlan Lake basin, Guatemala. Anales de Biología, (36), 23-31. https://doi.org/10.6018/analesbio.36.5

Gutiérrez, J. M. (2009). Snakebite Envenomation in Central America. En S. P. Mackessy (Ed.), Handbook of venoms and toxins of reptiles (pp. 492-505). Boca Raton, Florida: CRC Press.

Gutiérrez, J. M. (2014). Current challenges for confronting the public health problem of snakebite envenoming in Central America. Journal of Venomous Animals and Toxins including Tropical Diseases, 20(1), 7. https://doi.org/10.1186/16789199-20-7

Gutiérrez, J. M. (2019). Global availability of antivenoms: The relevance of public manufacturing laboratories. Toxins, 11(1). https:// doi.org/10.3390/toxins11010005

Gutiérrez, J. M., Calvete, J. J., Habib, A. G., Harrison, R. A., Williams, D. J., \& Warrell, D. A. (2017). Snakebite envenoming. Nature Reviews. Disease Primers, 3, 17063. https://doi.org/10.1038/ nrdp.2017.63

Gutiérrez, J. M., \& Sasa, M. (2002). Bites and envenomations by colubrid snakes in Mexico and Central America. Journal of Toxicology: Toxin Reviews, 21(1-2), 105-115. https://doi. org/10.1081/TXR-120004743

Hui Wen, F., Monteiro, W. M., Moura da Silva, A. M., Tambourgi, D. V., Mendonça da Silva, I., Sampaio, V. S., ... Lacerda, M. (2015). Snakebites and scorpion stings in the Brazilian Amazon: Identifying research priorities for a largley neglected problem. PLoS Neglected Tropical Diseases, 9(5), 1-11. https://doi.org/10.1371/ journal.pntd.0003701
Instituto Nacional de Estadística. (2002). Censos 2002: XI de población y VI de habitación. Guatemala: Autor.

Kasturiratne, A., Wickremasinghe, A. R., de Silva, N., Gunawardena, N. K., Pathmeswaran, A., Premaratna, R., ... de Silva, H. J. (2008). The global burden of snakebite: A literature analysis and modelling based on regional estimates of envenoming and deaths. PLoS Medicine, 5(11), 1591-1604. https://doi.org/10.1371/journal. pmed.0050218

Letona, C. A. (2012). Guía de animales ponzoñosos de Guatemala: Manejo del paciente intoxicado (Tesis de licenciatura). Universidad de San Carlos de Guatemala, Guatemala.

Lomonte, B. (2012). Revisión Venenos de serpiente: De la investigación al tratamiento. Acta Médica Costarricense, 54(2), 86-96.

Longbottom, J., Shearer, F. M., Devine, M., Alcoba, G., Chappuis, F., Weiss, D. J., ... Pigott, D. M. (2018). Vulnerability to snakebite envenoming: a global mapping of hotspots. The Lancet, 392(10148), 673-684. https://doi.org/10.1016/ S0140-6736(18)31224-8

López, M. A. (1993). Accidente ofídico: Estudio antropológico, clínico-epidemiológico, en el departamento de Sacatepéquez, enero de 1987 al 31 de diciembre de 1992. Guatemala (Tesis de licenciatura). Universidad de San Carlos de Guatemala, Guatemala.

Mackessy, S. P. (Ed.). (2009). Section III: Reptile Venom Enzymes. Handbook of venoms and toxins of reptiles. Boca Raton, Florida: CRC Press.

Maltez, J. C. (1994). Accidente ofídico: Estudio retrospectivo, clínico, antropológico y epidemiológico, realizado en el departamento de Guatemala, Región Sur, del 1 de enero de 1987 al 31 de diciembre de 1992, Guatemala (Tesis de licenciatura). Universidad de San Carlos de Guatemala, Guatemala.

Mazariegos, G. A. (1993). Accidente ofidico: Estudio antropológico, clínico-epidemiológico en el Departamento de Petén Occidental, del 1 de enero de 1987 al 31 de diciembre de 1992. Guatemala (Tesis de licenciatura). Universidad de San Carlos de Guatemala, Guatemala. 
McCleary, R. J. R., \& Kini, R. M. (2013). Snake bites and hemostasis/thrombosis. Thrombosis Research, 132(6), 642-646. https://doi.org/10.1016/j. thromres.2013.09.031

Mendoza, M. V. (1993). Accidente ofídico: Estudio retrospectivo, clínico, antropológico y epidemiológico, realizado en el Municipio de Amatitlán del 1 de enero 1987 al 31 de julio de 1993. Guatemala (Tesis de licenciatura). Universidad de San Carlos de Guatemala, Guatemala.

Menezes, M. C., Furtado, M. F., Travaglia-Cardoso, S. R., Camargo, A. C. M., \& Serrano, S. M. T. (2006). Sex-based individual variation of snake venom proteome among eighteen Bothrops jararaca siblings. Toxicon, 47(3), 304-312. https:// doi.org/10.1016/j.toxicon.2005.11.007

Ministerio da Saúde. (2001). Manual de Diagnóstico e Tratamento de Acidentes por Animais Peçonhentos (2. ${ }^{\mathrm{a}}$ ed.). Brasília: Autor.

Ministerio da Saúde. (2014). Guia de vigilância em saúde ( $2^{\mathrm{a}}$. ed. Vol. 1). Brasília: Autor.

Ministério da Saúde. (2019). Guia de vigilância em saúdes ( $3 .^{\mathrm{a}}$ ed.). Brasília: Autor. Recuperado de http://bvsms.saude.gov.br/bvs/publicacoes/guia vigilancia_saude_volume_unico_3ed.pdf

Muralles, C. J. (1993). Accidente ofídico: Estudio antropológico, clínico-epidemiológico, en el Departamento de El Progreso, enero de 1987 a diciembre de 1992. Guatemala (Tesis de licenciatura). Universidad de San Carlos de Guatemala, Guatemala.

Organización Panamericana de la Salud. (2009). Manual para la identificación, prevención $y$ tratamiento de mordeduras de serpientes venenosas en Centroamérica, Volumen I: Guatemala. Guatemala: Autor.

Overall, C. P. (1987). Toxicidad de los venenos de Bothrops asper y Bothrops nummifer de Guatemala en ratón blanco (Tesis de licenciatura). Universidad de San Carlos de Guatemala, Guatemala.

Pérez-Gómez, A. S., Monteiro, W. M., João, G. A. P., Sousa, J. D. de B., Safe, I. P., Damian, M. M., ... Silva, I. M. da. (2019). Hemorrhagic stroke following viper bites and delayed antivenom administration: three case reports from the
Western Brazilian Amazon. Revista da Sociedade Brasileira de Medicina Tropical, 52, e20190115. https://doi.org/10.1590/0037-8682-0115-2019

Ramos, J. R. (1994). Cuadro clínico y hallazgos anatomopatológicos en accidente ofídico (Tesis de licenciatura). Universidad de San Carlos de Guatemala, Guatemala.

Rojas, E., Saravia, P., Angulo, Y., Arce, V., Lomonte, B., Chávez, J. J., ... Gutiérrez, J. M. (2001). Venom of the crotaline snake Atropoides nummifer (jumping viper) from Guatemala and Honduras: comparative toxicological characterization, isolation of a myotoxic phospholipase A2 homologue and neutralization by two antivenoms. Comparative Biochemistry and Physiology Part C Toxicology \& Pharmacology, 129, 151-162. https://doi.org/10.1016/S1532-0456(01)00198-3

Rojas, G., Gutiérrez, J. M., Gené, J. A., \& Gómez, M. (1987). Neutralización de las actividades tóxicas y enzimáticas de cuatro venenos de serpientes de Guatemala y Honduras por el antiveneno polivalente producido en Costa Rica. Revista de Biología Tropical, 35(1), 59-67.

Saravia-Otten, P., Hernandez, R., Gutiérrez, J. M., Mérida, M., \& Cáceres, A. (2015). Evaluación de la capacidad neutralizante de extractos de plantas de uso popular en Guatemala como antídotos para el envenenamiento por la mordedura de Bothrops asper. Ciencia, Tencología y Salud, 2(1), 25-37.

Saravia-Otten, P., Hernández, R., Marroquín, N., García, G., \& Mérida, M. (2017). Inhibición de los efectos coagulante, fosfolipasa A 2 y proteolítico del veneno de Bothrops asper por plantas usadas tradicionalmente en Centroamérica. Revista Ciencia, Tecnología y Salud, 4(2), 203-216.

Saravia, P., Rojas, E., Escalante, T., Arce, V., Chaves, E., Velásquez, R., ... Gutiérrez, J. M. (2001). The venom of Bothrops asper from Guatemala: Toxic activities and neutralization by antivenoms. Toxicon, 39(2-3), 401-405. https://doi.org/https:// doi.org/10.1016/s0041-0101(00)00122-77

Silva de Oliveira, S., Campos Alves, E., dos Santos Santos, A., Freitas Nascimento, E., Tavares Pereira, J. P., Mendonça da Silva, I., ... Monteiro, W. M. (2019). Bothrops snakebites in the Amazon: Recovery from hemostatic disorders after Brazilian antivenom therapy. Clinical 
Toxicology, 58(4), 1-9. https://doi.org/10.1080/1 5563650.2019 .1634273

Squaiella-Baptistão, C. C., Sant'Anna, O. A., Marcelino, J. R., \& Tambourgi, D. V. (2018). The history of antivenoms development: Beyond Calmette and Vital Brazil. Toxicon, 150, 86-95. https://doi. org/10.1016/j.toxicon.2018.05.008

Uetz, P., Freed, P. \& Hošek, J. (s. f.). The Reptile Database. Recuperado 26 de julio de 2019, de http://reptile-database.reptarium.cz/ search?search $=$ guatemala + snakes\&submit=Search Valdés, A. M., Chuy, S., Lara, O., Torres, P., Escobedo, C., \& Ibarra, G. (1994). Venenos y serpientes venenosas y ofidismo en Guatemala. Revista, 10(1), 16-19.

Velásquez, A. E. (1993). Accidente ofidico: Estudio antropológico, clínico, epidemiológico, en el departamento de Santa Rosa, del 1 de enero de 1987 al 31 de diciembre de 1992. Guatemala (Tesis de licenciatura). Universidad de San Carlos de Guatemala, Guatemala.
World Health Organization. (2019). Snakebite envenoming: A strategy for prevention and control. Geneva: Autor.

Yañez-Arenas, C., Townsend Peterson, A., RodríguezMedina, K., \& Barve, N. (2016). Mapping current and future potential snakebite risk in the new world. Climatic Change, 134(4), 697-711. https:// doi.org/10.1007/s10584-015-1544-6

Yee-Seuret, S., Vargas-González, A., \& HernándezTorres, L. (2012). Mordeduras por serpientes venenosas en Guatemala. Revista Electrónica de Portales Medicos.com. Recuperado de https:// www.portalesmedicos.com/publicaciones/ articles/4946/1/Mordeduras-por-serpientesvenenosas-en-Guatemala.html

Zamuner, S. R., Zuliani, J. P., Fernandes, C. M., Gutiérrez, J. M., \& Pereira Teixeira, C. D. F. (2005). Inflammation induced by Bothrops asper venom: Release of proinflammatory cytokines and eicosanoids, and role of adhesion molecules in leukocyte infiltration. Toxicon, 46(7), 806-813. https://doi.org/10.1016/j.toxicon.2005.08.011 